\title{
Morphological and molecular characteristics of adult worms of Gnathostoma Owen, 1836 (Nematoda) collected from domestic pigs in Dien Bien Province, northern Vietnam
}

\author{
Nguyen Van Tuyen ${ }^{1}$, Nguyen Thi Kim Lan ${ }^{2}$, Pham Ngoc Doanh ${ }^{3,4}$ \\ ${ }^{1}$ Dien Bien Technical Economic College, Dien Bien Province, Vietnam; \\ ${ }^{2}$ Thai Nguyen University of Agriculture and Forestry, Vietnam; \\ ${ }^{3}$ Institute of Ecology and Biological Resources, Vietnam Academy of Science and Technology (VAST), Hanoi, Vietnam; \\ ${ }^{4}$ Graduate University of Science and Technology, VAST
}

\begin{abstract}
Gnathostomes are of interest because of their unique appearance and medical importance. Among 13 valid species of the genus Gnathostoma Owen, 1836 (Nematoda: Spirurida), two species, G. doloresi Tubangui, 1925 and G. hispidum Fedtschenko, 1872 , are parasites of pigs but their larvae can infect humans to cause gnathostomiasis. In this study, we collected adults of Gnathostoma sp. from the stomach of domestic pigs (Sus scrofa domesticus Linnaeus) from Dien Bien Province, northern Vietnam. Morphologically, nematodes found here are most similar to $G$. doloresi with a slight difference in the spicules of males. In contrast, they are genetically distinct from G. doloresi and other species of Gnathostoma in both ITS2 and cox 1 sequences. The findings of the present study suggest that specimens of Gnathostoma sp. found in Dien Bien are likely a new species and emphasise the need of further studies on the taxonomy and phylogenetic relationship of species of Gnathostoma. Special attention should also be paid to swine and human gnathostomiasis in Dien Bien Province, Vietnam and the neighbouring areas of China and Laos.
\end{abstract}

Keywords: Gnathostomatidae, morphology, cox1, ITS2, South-East Asia.

Nematodes of the genus Gnathostoma Owen, 1836 are parasites of mammals, particularly carnivores, marsupials and pigs (Miyazaki 1991, Nawa et al. 2015). The advanced third-stage larvae of some species, such as $G$. binucleatum Almeyda-Artigas, 1991, G. doloresi Tubangui, 1925, G. hispidum Fedtschenko, 1872, G. nipponicum Yamaguchi, 1941 and G. spinigerum Owen, 1836, can infect humans causing a zoonotic disease, gnathostomiasis.

The most common manifestations of human infection are migratory swellings under the skin and peripheral blood eosinophilia. In addition, larvae of Gnathostoma spp. can migrate into other sites such as the liver to cause abscesses, into the eye, resulting in visual loss, or into the peripheral or central nervous system including spinal cord and brain, resulting in nerve pain, paralysis, coma and death (Waikagul and Diaz-Camacho 2007, Nawa et al. 2015). Given the medical importance, species of Gnathostoma have been intensively studied (Nawa et al. 2015).

So far, more than twenty species of Gnathostoma have been described as parasites of mammals, mainly in Asia and the Americas (Bertoni-Ruiz et al. 2011), of which 13 species are considered to be valid (Waikagul and Diaz-Camacho 2007, Nawa et al. 2015). Among them, two species,
G. doloresi and G. hispidum, are parasites of domestic pigs and wild boars but their larvae can infect humans (Waikagul and Diaz-Camacho 2007, Nawa et al. 2015).

Although these two species have been reported from Vietnam (Le et al. 1996), there is a lack of the morphological and molecular data on these medically important swine parasites. Recently, while conducting an epidemiological survey on pig diseases, we found adult worms of Gnathostoma in the stomach of domestic pigs (Sus scrofa domesticus Linnaeus) from Dien Bien Province, northern Vietnam, where free-range pig production is common. The present paper describes the morphological and molecular characteristics of these specimens collected in Vietnam.

\section{MATERIALS AND METHODS}

A total of 50 stomachs of domestic pigs were purchased from local pig farmers in Dien Bien Province, northern Vietnam, for parasite examination. Adults of Gnathostoma sp. were collected from five (10\%) out of 50 pigs. The worms were washed in physiological saline to remove food debris and mucus and fixed in $10 \%$ formalin for morphological study. Four worms (two males and two females) were fixed separately in $96 \%$ ethanol for molecular analyses. For morphological examination, sixteen adult worms

Address for correspondence: Pham Ngoc Doanh, Department of Parasitology, Institute of Ecology and Biological Resources, Vietnam Academy of Science and Technology, Hanoi, Vietnam. E-mail: pndoanh@yahoo.com 
A

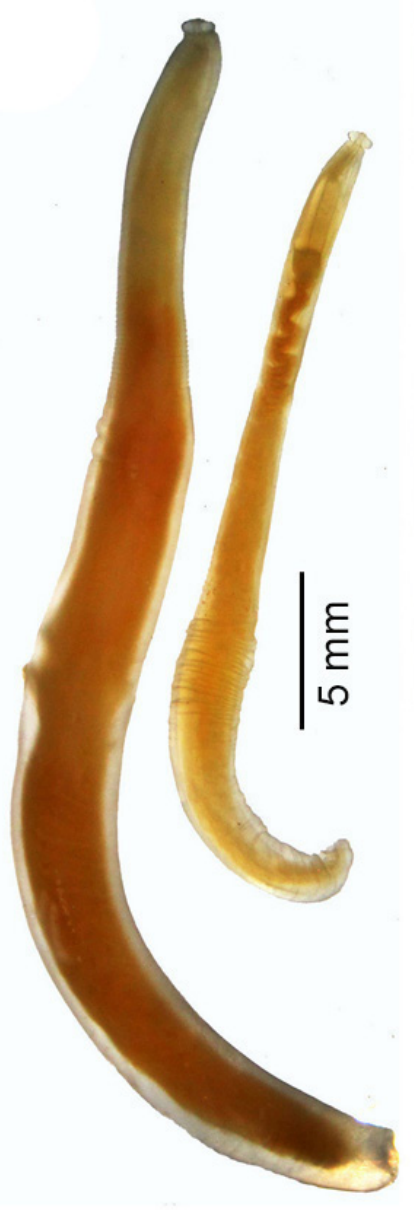

B
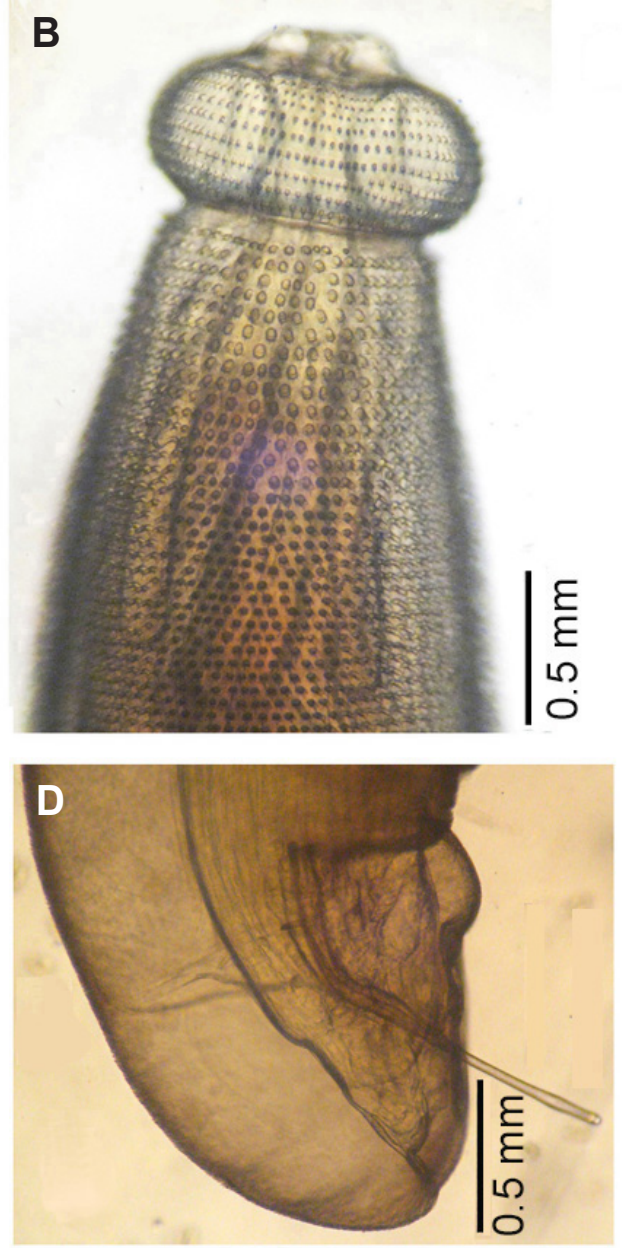
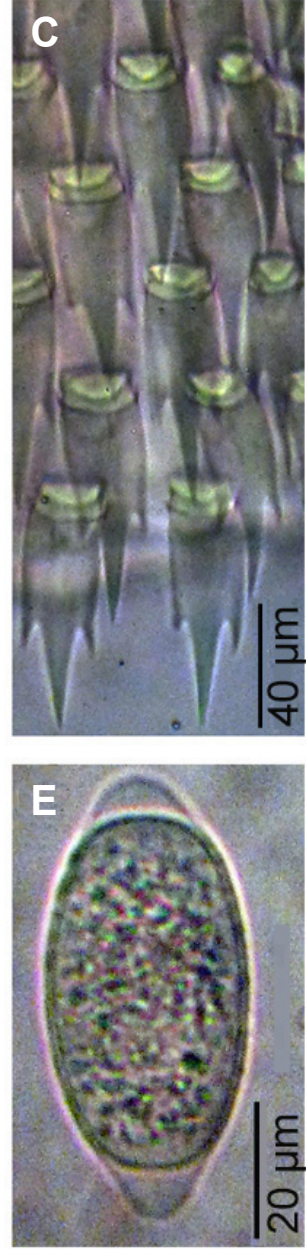

Fig. 1. Gnathostoma sp. collected from the stomach of pigs in Dien Bien Province, Vietnam. A - whole body of a female (left) and a male (right); B - anterior part of a male; $\mathbf{C}$ - region of tridentate spines; $\mathbf{D}$ - posterior part of a male showing two unequal spicules; $\mathbf{E}$ - egg with a polar bulge on both ends.

fixed in formalin were cleared in lactophenol for morphological and morphometric study. The worms were examined under a stereomicroscope and an Axio Lab A1 microscope (Carl Zeiss, Oberkochen, Germany).

For molecular analyses, genomic DNA from individual worms was extracted using a QIAamp DNA Stool Mini Kit (Qiagen, Hilden, Germany). The internal transcribed spacer 2 (ITS2) region of nuclear ribosomal DNA and a partial region of the mitochondrial cytochrome $\mathrm{C}$ oxidase subunit 1 ( $\operatorname{cox} 1)$ were chosen to analyse because these genetic markers were used for identification of species of Gnathostoma (see Ando et al. 2006).

ITS2 region was amplified using polymerase chain reaction (PCR) with the primer pair NEWS2 and ITS2-RIXO (Almeyda-Artigas et al. 2000). A part of the cox 1 gene was amplified using PCR with the primer pair JB3 and JB4.5 (Bowles et al. 1993). PCR products were purified using a Qiaquick PCR Purification Kit (Qiagen). These products were primed using a Big-Dye Terminator Cycle Sequencing Kit and both strands were directly sequenced using an Ab3730 sequencer (Applied Biosystems, Foster City, CA, USA), using the PCR primers as sequencing primers.

Eight nucleotide sequences of the ITS2 and $\operatorname{cox} 1$ gene from four samples were obtained and deposited in the DNA data bank of Japan (DDBJ) with the accession numbers LC404124LC404127 (cox1 sequences) and LC390038-LC390041 (ITS2 sequences). In addition, ITS2 and cox 1 sequences of other species of Gnathostoma were obtained from the GenBank nucleotide database for analyses and for reconstruction of phylogenetic trees in MEGA6 using the Maximum likelihood method based on the best model (Tamura et al. 2013).

\section{RESULTS AND DISCUSSION}

The result of the morphological examination showed that the body of specimens of Gnathostoma sp. collected from pigs in Dien Bien is slightly widened posteriorly, mature females $(30-42 \times 2.4-4 \mathrm{~mm}$ in size $)$ are bigger than males $(22-25 \times 1.8-2.2 \mathrm{~mm})($ Fig. 1A). The head bulb is armed with 9-11 rows of hooklets (Fig. 1B). The whole body is covered with cuticular spines which vary in the number of teeth: multi-dentate (4-6 teeth), tridentate ( 3 teeth), bidentate ( 2 teeth) and unidentate spines. Of these, the tridentate spines occupy a major part of the body (Fig. 1C). The unidentate region includes single-pointed spines with a gradual change of the size to more slender and hair-like spines towards the posterior end of the worms.

The tail of the males curves ventrally. Spicules of males are unequal, with blunt apex (Fig. 1D). Longer spicule measures $1.1-1.4 \mathrm{~mm}$ in length; shorter spicule is $0.6-0.7 \mathrm{~mm}$ long. 


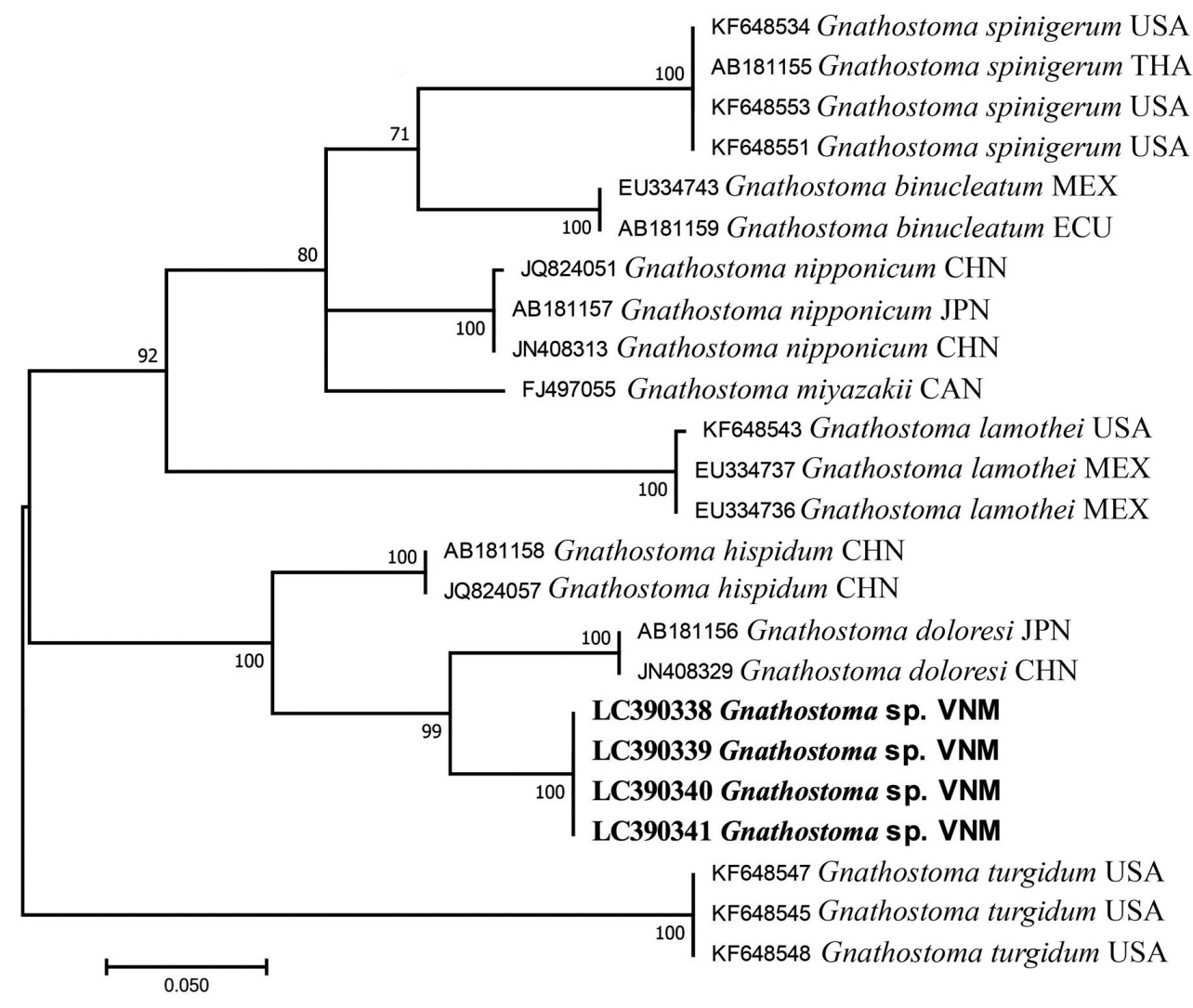

Fig. 2. Molecular phylogenetic tree reconstructed from ITS2 sequences using the Maximum Likelihood method based on the Kimura 2-parameter model. The bootstrap values are shown next to the branches. A discrete Gamma distribution was used to model evolutionary rate differences among sites. The tree is drawn to scale, with branch lengths measured in the number of substitutions per site. All positions containing gaps and missing data were eliminated. The nucleotide sequences obtained in this study are printed in bold and others from the DNA database are shown with Accession No. and country code: CAN - Canada, CHN - China, ECU-Ecuador, JPN Japan, MEX - Mexico, THA - Thailand, USA - United States of America, VNM - Vietnam.

The tail of females is blunt (Fig. 1A). Eggs are $64-70 \times 30-32 \mu \mathrm{m}$ in size, with two polar bulges (Fig. 1E). In these characteristics, specimens from Vietnam are similar to Gnathostoma doloresi (see Miyazaki 1991).

In contrast, the molecular data showed that Gnathosto$m a$ sp. of this study was genetically distinct from G. doloresi as well as the other congeners in both ITS2 and cox 1 sequences. For ITS2, the nucleotide differences of Gnathostoma sp. from other Gnathostoma species were high: $8.6 \%$ vs G. doloresi, $12.9 \%$ vs G. hispidum and 26.5-31.6\% vs other species, namely, G. nipponicum, G. miyazaki Anderson, 1964, G. binucleatum, G. lamothei Bertoni-Ruiz, Garcia-Prieto, Osorio-Sarabia et Léon-Règagnon, 2005, G. turgidum Stossich, 1902 and G. spinigerum. In the phylogenetic tree (Fig. 2), samples of Gnathostoma sp. made a distinct group close to G. doloresi and G. hispidum.

For $\operatorname{cox} 1$ sequences, the nucleotide differences between samples of Gnathostoma sp. and other species were also high $(14.2-14.9 \%$ vs $G$. binucleatum, $14.3-18.1 \%$ vs G. spinigerum, $16.7-18.5 \%$ vs G. hispidum, 17.2-20.1\% vs G. doloresi and $21.5-22.9 \%$ vs G. nipponicum). In the phylogenetic tree, every species formed a separate clade. Gnathostoma sp. was markedly distant from G. doloresi and grouped with G. hispidum, but with a low bootstrap value (Fig. 3).
For traditional morphological identification of adults species of Gnathostoma, the most important characteristics are the pattern of cuticular spines and the number of polar bulges of eggs (Miyazaki 1991, Nawa et al. 2015). Till now, only two species, G. doloresi and G. hispidum, were found in the stomach of pigs and wild boars (Bertoni-Ruiz et al. 2011, Nawa et al. 2015). Both species have cuticular spines covering the whole body, but they are different from one another in the patterns of the cuticular spines and the number of polar bulges of eggs. Eggs of G. hispidum have only one bulge, whereas those of $G$. doloresi have a bulge at both ends (Miyazaki 1991, Nawa et al. 2015).

Gnathostoma sp. collected in Dien Bien Province, Vietnam, is most similar to G. doloresi in two important characteristics: the presence of spines on the whole body surface and bipolar-bulge eggs. They also show similarities in the pattern of cuticular spines. The difference between them is the ratio of long/short spicules of males. They both have a similar short spicule (0.6-0.7 $\mathrm{mm}$ in length), but the length of the long spicule of Gnathostoma sp. reported here $(1.1-1.4 \mathrm{~mm})$ is much shorter than that $(1.9-2.1 \mathrm{~mm})$ of G. doloresi (see Maplestone 1930).

In contrast to morphological similarity, specimens of Gnathostoma sp. are clearly distinct genetically from G. doloresi and the other congeners in both ITS2 and cox 1 sequences. As can be seen in the phylogenetic trees 


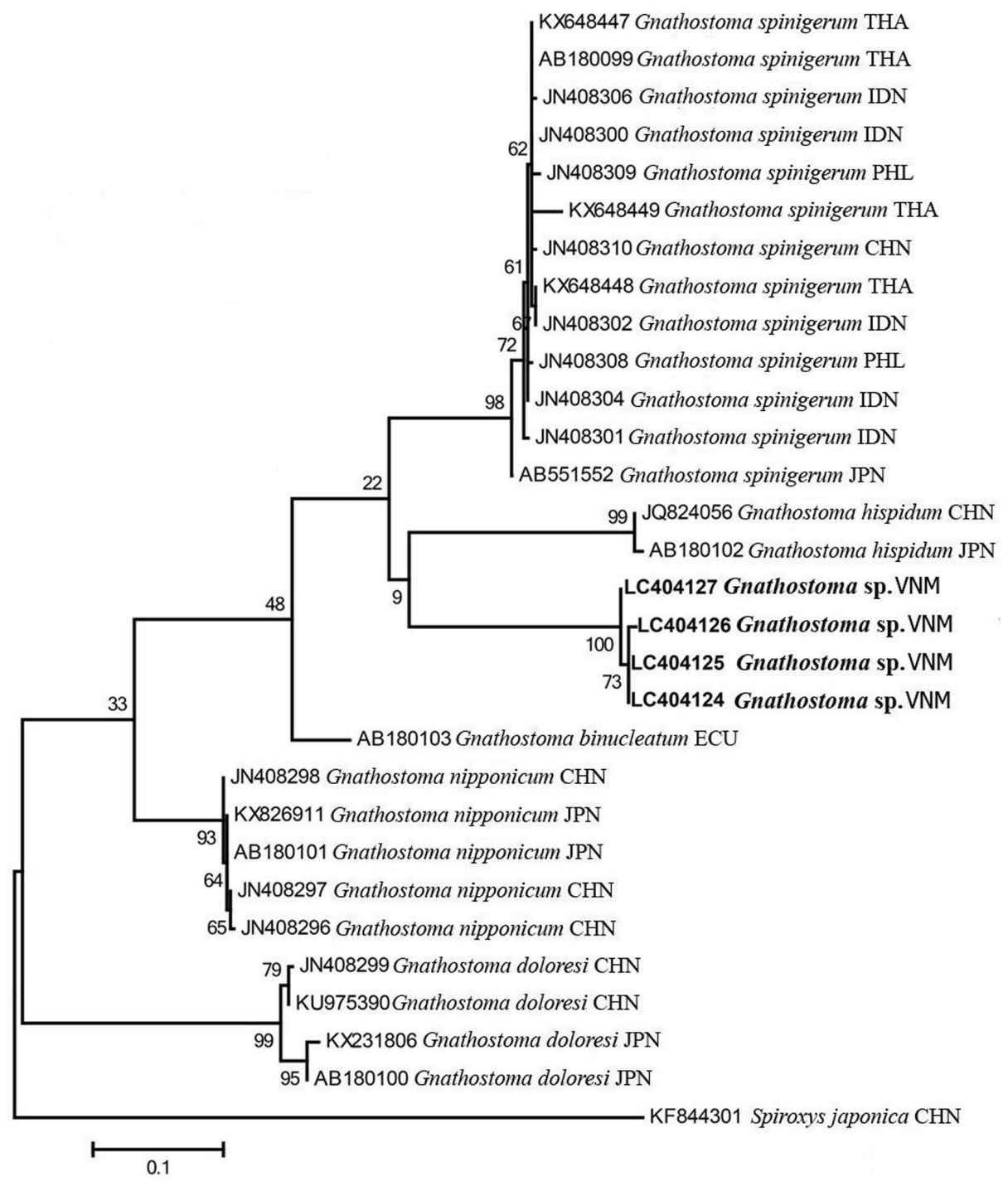

Fig. 3. Molecular phylogenetic tree of species of Gnathostoma reconstructed from cox 1 sequences using the Maximum Likelihood method. The evolutionary history was inferred using the Maximum Likelihood method based on the Hasegawa-Kishino-Yano model. The bootstrap values are shown next to the branches. A discrete Gamma distribution was used to model evolutionary rate differences among sites. The tree is drawn to scale, with branch lengths measured in the number of substitutions per site. All positions containing gaps and missing data were eliminated. The nucleotide sequences obtained in this study are printed in bold and others from DNA database are shown with Accession No. and country code: CHN - China, ECU-Ecuador, IDN - Indonesia, JPN - Japan, PHL - Philippines, THA - Thailand VNM - Vietnam.

(Figs. 2, 3), G. doloresi from two different countries, China and Japan, is identical (ITS2) or very similar ( $\operatorname{cox} 1)$. In contrast, Gnathostoma sp. from Vietnam belongs to a distinct group far distant from G. doloresi. Molecular data thus suggest that Gnathostoma sp. most likely represents a distinct species.

At present, it is difficult to find out any clear-cut morphological differences between Gnathostoma sp. and G. doloresi, because the morphological descriptions of $G$. dolore$s i$ were not detailed enough. The original description by Tubangui (1925) was very simple without description of the shape and size of cuticular spines. Eduardo (1989) described the spines of $G$. doloresi from the Philippines but did not mention the presence of bidentate spines. Koga and Ishii (1992) described body spines of $G$. doloresi from Japan including all four regions, but bidentate and tridentate spines were reported as narrower compared to those of $G$. doloresi from the Philippines.

Since the pattern of cuticular spines is an important taxonomic characteristic for identification of Gnathostoma spp., more detailed descriptions of the body spines as well as other characteristics, especially of females $G$. doloresi and Gnathostoma sp., are necessary. ITS2 and cox 1 sequences have been commonly used for species identification and phylogenetic analyses of species of Gnathostoma (e.g. Almeyda-Artigas et al. 2000, Bertoni-Ruiz et al. 2005, Martínez-Salazar et al. 2005, Ando et al. 2006, Hernández-Gómez et al. 2010, Li et al. 2015, Jongthawin et al. 2016).

However, some lineages were not strongly supported in the phylogenetic trees, especially in the cox 1 tree, generated in the present study. More sequence data on Gnathostoma spp. are, therefore, necessary to obtain a better res- 
olution of phylogenetic relationships among the members of this genus. Since two previously known species from swine, G. hispidum and G. doloresi, are zoonotic parasites that affect the health of pigs and humans, special attention should be paid to swine and human gnathostomiasis in Dien Bien Province, Vietnam and the neighbouring areas of China and Laos.

In conclusion, Gnathostoma sp. of specimens collected from the stomachs of pigs in Dien Bien Province, northern Vietnam, are morphologically similar to $G$. doloresi with a slight difference in spicules of males, but are genetically far distant from $G$. doloresi and other congeners, representing a putative new Gnathostoma species. Further studies on the taxonomy and phylogenetic relationship of species of Gnathostoma are required to find out more taxonomic characteristics for their identification.

\section{Ethical approval}

The present study was approved by the Rector of Thai Nguyen University of Agriculture and Forestry (decision no. 225/QD-DHNL-DT dated February 28, 2018). Stomachs of domestic pigs were purchased from the local owners in Dien Bien Province where pork meat and visceral parts of pigs are sold in local markets. The use of the stomachs for research purpose was informed to the owners with their agreement.

\section{REFERENCES}

Almeyda-Artigas R.J., Bargues D.M., Mas-Coma S. 2000 ITS2 rDNA sequencing of Gnathostoma species (Nematoda) and elucidation of the species causing human gnathostomiasis in the Americas. J. Parasitol. 86: 537-544.

Ando K., Tsunemori M., Akahane H., Tesana S., Hasegawa H., Chinzei Y. 2006: Comparative study on DNA sequences of ribosomal DNA and cytochrome c oxidase subunit 1 of mitochondrial DNA among five species of gnathostomes. J. Helminthol. 80: 7-13.

Bertoni-Ruiz F., Lamothe-Argumedo M.R.L., García-Prieto L., Osorio-Sarabia D., León-Règagnon V. 2011: Systematics of the genus Gnathostoma (Nematoda: Gnathostomatidae) in the Americas. Rev. Mex. Biodivers. 82: 453-464.

Bertoni-Ruiz F., García-Prieto L., Osorio-Sarabia D., LEÓN-RÈGAGNON V. 2005: A new species of Gnathostoma (Nematoda: Gnathostomatidae) in Procyon lotor hernandezil from Mexico. J. Parasitol. 91: 1143-1149.

Bowles J., Hope M., Tiu W.U., Liu S.X., McManus D.P. 1993 Nuclear and mitochondrial genetic markers highly conserved between Chinese and Philippine Schistosoma japonicum. Acta Trop. 55: 217-229.

EduARDo S.L. 1989: Scanning electron microscopy of the integumental surfaces of adult Gnathostoma doloresi Tubangui, 1925, a parasite of pigs in the Philippines. Trans. Nat. Aca. Sci. Tech. (Phils.). 11: 97-102.

Hernández-Gómez R.E., Martínez-Salazar E.A., LóPEZ-JiménEZ S., León-RÈGagnon V. 2010: Molecular identification of the advanced third-stage larvae of Gnathostoma lamothei in Tabasco, Mexico. Parasitol. Int. 59: 97-99.

Jongthawin J., Intapan P.M., Sanpool O., Janwan P., SadaOw L., Thanchomnang T., Laymanivong S., Maleewong W. 2016: Molecular phylogenetic confirmation of Gnathostoma spinigerum Owen, 1836 (Nematoda: Gnathostomatidae) in Laos and Thailand. Folia Parasitol. 25: 63.
Koga M., IsHiI Y. 1992: Surface topography of adults and eggs of Gnathostoma doloresi (Nematoda: Spirurida) from wild boars (Sus scrofa leucomystax). J. Helminthol. Soc. Wash. 59: 83-88.

LE N.T. (ED.) 1996: [Helminths of Domestic Animals in Vietnam.] Science and Technics Publishing House, Hanoi, 296 pp. (In Vietnamese.)

Li W.W., Ren Y.J., Li J., Huang W.Y. 2015: Scanning electron microscopic observation on adult Gnathostoma doloresi worms and the phylogenetic analysis of $G$. doloresi based on ITS2 and coxl gene sequences. Chin. J. Parasitol. Parasit. Dis. 33: 130134.

Maplestone H.P.A. 1930: Nematode parasites of pigs in Bengal. Rec. Ind. Mus. 32: 77-105.

Martínez-Salazar E.A., León-RÈGagnon V. 2005: Confirmation of Gnathostoma binucleatum Almeyda-Artigas, 1991, advanced third-stage larvae in Tres Palos Lagoon, Mexico, by morphological and molecular data. J. Parasitol. 91: 962-965.

Miyazaki I. (Ed.) 1991: An Illustrated Book of Helminthic Zoonoses. International Medical Foundation of Japan, Fukuoka, 494 pp.

Nawa Y., Intapan P.M., Maleewong W., Diaz-Camacho S.P. 2015: Gnathostoma. In L. Xiao, U. Ryan, Y. Feng (Eds), Food Microbiology Series: Biology of Foodborne Parasites. New York, CRC Press, pp. 405-426.

Tamura K., Stecher G., Peterson D., Filipski A., Kumar S. 2013: MEGA6: Molecular Evolutionary Genetics Analysis version 6.0. Mol. Biol. Evol. 30: 2725-2729.

Tubangui M.A. 1925: Metazoan parasites of Philippine domestic animals. Philipp. J. Sci. 28: 11-38.

Waikagul J., Diaz-Camacho S.P. 2007: Gnathostomiasis. In K.D. Murrell and B. Fried (Eds), Food-Borne Parasitic Zoonoses. Springer, New York, pp. 235-226. 010 . 